\title{
Neutron Reflectometry of an Anionic Surfactant at the Solid-Liquid Interface under Shear
}

\author{
Rebecca J. L. Welbourn, ${ }^{*}{ }^{\dagger}$ Felicity Bartholomew, ${ }^{\dagger}$ Philipp Gutfreund, ${ }^{\dagger}$ and Stuart M. Clarke ${ }^{\dagger}$ (i) \\ ${ }^{\dagger}$ BP Institute and Department of Chemistry, University of Cambridge, Madingley Rise, Madingley Road, Cambridge, U.K. \\ ${ }^{*}$ Institute Laue-Langevin, Grenoble, France
}

\section{Supporting Information}

ABSTRACT: Neutron reflectometry with in situ rheology is used to measure the shear response of an adsorbed anionic surfactant (sodium bis(2-ethylhexyl) sulfosuccinate, AOT) at the alumina-water interface. A low surfactant concentration is measured where a single bilayer adsorbs at the interface as well as a higher concentration where a multilamellar structure forms. The low concentration structure does not change with the imposed shear (oscillatory or steady). However, the lamellar phase shows a loss of structure under both steady and oscillatory shear. There are differences between the steady and oscillatory cases, which are discussed, with both showing a strong dependence on the strain amplitude.

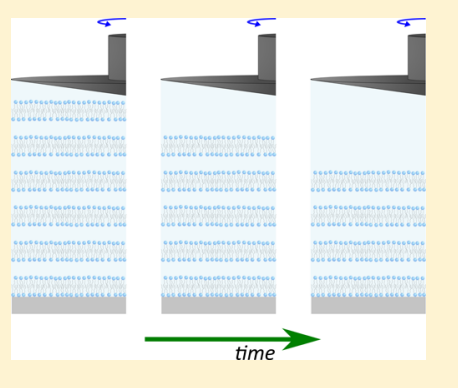

\section{INTRODUCTION}

There are many applications where the flow of fluid against a solid surface is important, such as flow in a pipe or with lubricants. Organic molecules are often present in these systems as they have been found to enhance the performance; this may be by changing the flow itself (e.g., wetting behavior) or through more indirect methods (e.g., corrosion inhibition). In both cases it is interesting to determine what, if any, effect the flow has on these adsorbed molecules. It is experimentally very challenging to characterize these molecules and any changes at the solid/liquid interface. Within this work, neutron reflectometry (NR) is used as a probe of this buried interface to try and improve understanding of how shear flow alters these species on a molecular scale.

When a system is subject to an applied shear, the effects will depend on the relative shear rates applied. For example, the flow of fluid in a pipe occurs at a relatively low shear rate of $10^{2}$ $\mathrm{s}^{-1}$, whereas shear of fluids in oil recovery occur at much slower rates. In contrast, much higher rates, $10^{6} \mathrm{~s}^{-1}$, are found in the gap between the camshaft and follower of an engine. Within this work, rates typical of pipe flow are considered. Methods to access high shear rates are being developed.

The lubricating ability of a solution on a surface is generally tested by comparing the coefficients of friction combined with rheology to help understand the bulk changes. There is also considerable evidence that shear flow can change bulk phase behavior and particularly that orientational ordering is affected. $^{1-7}$ The usual interpretation of this behavior is that the shear causes the surfactant to favor a phase which is not the stable phase when quiescent. Generally, this change is found to occur when the system is reasonably close to the phase boundary.

While it is useful to understand the changes within the bulk solution, it is also important to understand interfacial changes which will help to fully explain the observed lubricating ability of a system. These changes occur on a molecular level and require different experimental methods. NR is used here as it is sensitive to changes in composition of layers at interfaces on a molecular scale. By using a rheometric cell, the effect of applied shear on adsorbed surface layers at the solid-liquid interface can be probed in situ.

A similar combination of rheology and NR has previously been applied to a range of high concentration polymer systems at the silicon-water interface and slip of hexadecane on silicon by Wolff et al..$^{8-10}$ as well as the adsorption of asphaltenes onto hydrophobic Si by Corvs et al. ${ }^{11}$ A crystal of silicon was used as the "plate" in a cone-plate rheometer setup, with the neutron beam reflected up through this solid substrate to the solidliquid interface. This is schematically illustrated in Figure 1.

An alternative method for measuring the effect of shear at the solid-liquid interface is to use a flow cell to produce laminar flow (Poiseuille shear) adjacent to the solid surface of interest. This has been successfully employed by Penfold et al. ${ }^{12}$ to characterize a multilayer of nonionic surfactant at the Si-water interface, which became more ordered on application of a shear gradient above $10^{4} \mathrm{~s}^{-1}$. Conversely, van der Grinten et al. ${ }^{13}$ found that polystyrene coated onto $\mathrm{Si}$ was partially removed by a critical shear rate between 3300 and $1 \times 10^{4} \mathrm{~s}^{-1}$. These observations suggest that different forces hold the surfactant and polymer near the surface in these two systems. More recently, a similar flow cell was used to simulate the effect of "rinsing" in surfactant applications at the air-liquid interface, with flow of a lower concentration through the bottom of the cell. ${ }^{14}$ Additional studies have used a Poiseuille cell to measure near-surface bulk behavior using grazing incidence small-angle neutron scattering (GISANS), with NR for mixed surfactant

Received: February 15, 2017

Revised: $\quad$ May 1, 2017

Published: May 22, 2017 


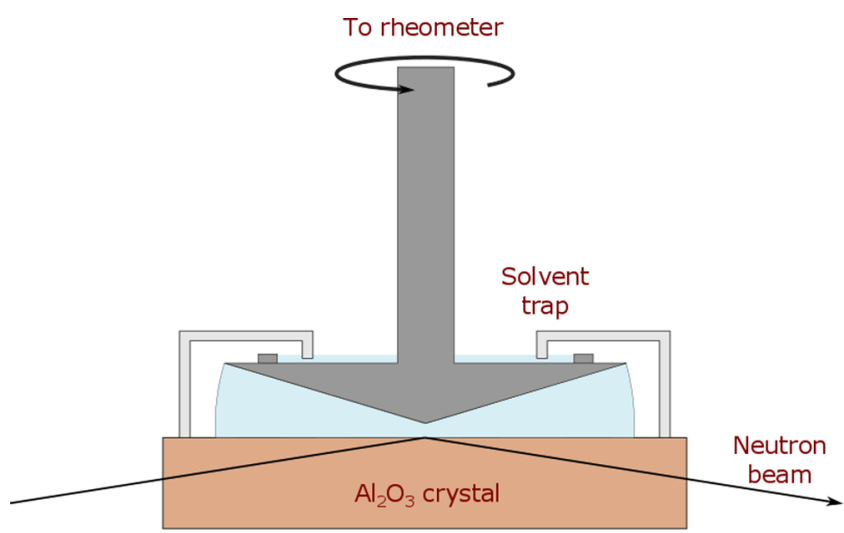

Figure 1. Illustration of the rheometric cone/plate geometry, with the incident and reflected neutron beams. The cone angle has been greatly exaggerated.

systems, with some differences between the surface and bulk..$^{15-17}$

The effect of confinement on additives can also be considered using surface forces apparatus (SFA), which has been used to measure the resistive force between two surfaces brought into contact. Because of the requirement for atomically flat surfaces, there has been some limitation in the range of experiments performed; however, Graca et al. ${ }^{18}$ have shown how this can be expanded by grafting a polymer monolayer onto each surface. Kuhl et al. ${ }^{19}$ have also developed the SFA measurements to consider this confinement in situ with NR, where they again consider the effects on grafted polymer brushes. An alternative form of confinement cell has been developed by Richardson et al. using an inflated diaphragm to apply an even pressure and narrow solution gap with $\mathrm{NR}^{20,21}$ These approaches provide complementary information to the other shear studies.

In this work a combined rheometer and NR experiment is used to monitor changes in an adsorbed surfactant at the solidliquid interface under shear. The system has been chosen to a provide order over a range of length scales which may be affected by different shear regimes: the surfactant sodium bis(2ethylhexyl) sulfosuccinate (AOT) at the alumina-water interface. The adsorption behavior under static conditions of this system is reasonably well documented as well as the bulk solution behavior. Hellsing et al. have reported that AOT forms a $35 \AA$ thick bilayer at the alumina-water interface at low concentrations and multilamellar (100s of $\mathrm{nm}$ ) structure at higher concentrations (approximately 2 wt \% or $50 \mathrm{mM}$ ) using $\mathrm{NR}^{22,23}$ The multilayers were identified through the presence of two orders of Bragg peaks, caused by interference of the scattering from these repeating layers. A similar surface bilayer has been found at the Si-water and air-water interfaces, with slight differences in thickness and separation. ${ }^{24-26}$ In a recent review of multilayers at the air-water interface, Thomas and Penfold describe how a swollen lamellar phase of AOT forms at the interface with slightly smaller $d$-spacing than in the bulk lamellar phase. ${ }^{24}$ Based on this characterization, essentially the same phase is found in all the AOT multilayer literature systems discussed.

The micellar and lamellar phases have also been wellcharacterized in solution, ${ }^{25-28}$ with some additional experiments carried out under shear for the higher AOT concentrations. One study reports viscoelastic behavior, with shear thickening and possible multilamellar vesicles (MLVs) forming at concentrations $3<$ wt $\%<18.5 .^{29}$ Higher concentrations still showed a weak gel, which was disrupted by addition of $\mathrm{NaCl}^{30,31}$ Bergenholtz and Wagner considered the kinetics of MLV formation at 17 wt \% AOT in brine and found differences under controlled stress versus controlled strain. $^{3}$ At 7 wt \% AOT concentration, Leon et al. ${ }^{5}$ found varying planar and $\mathrm{MLV}$ phases as the $\mathrm{NaCl}$ concentration was varied under shear. Clearly the effect of shear on bulk lamellar surfactant phases is quite complex, but phase changes can be identified by large changes in the viscosity of the solution. This can be helpful in providing context for changes at the solidliquid interface.

\section{EXPERIMENTAL SECTION}

Sodium bis(2-ethylhexyl) sulfosuccinate (AOT), $\mathrm{NaCl}$, and $\mathrm{D}_{2} \mathrm{O}$ were all obtained from Sigma-Aldrich and used as supplied. $\mathrm{All} \mathrm{H}_{2} \mathrm{O}$ was prepared from a Millipore system with a conductivity of $18.2 \mathrm{M} \Omega \cdot \mathrm{cm}$. Solutions of $2.5 \mathrm{mM}$ (CMC) or $50 \mathrm{mM}$ AOT were made up by mass in $\mathrm{H}_{2} \mathrm{O}$ and $\mathrm{D}_{2} \mathrm{O}$ as required. The alumina single crystals (sapphire (0001)) for the NR measurements were purchased from PiKem and polished by the supplier to a very low surface roughness, quoted as $\leq 5$ $\AA$ roughness $(\mathrm{RMS})(70 \times 70 \times 15 \mathrm{~mm})$. These were cleaned with a mild piranha solution $\left(5: 4: 1 \quad \mathrm{H}_{2} \mathrm{O}: \mathrm{H}_{2} \mathrm{SO}_{4}: \mathrm{H}_{2} \mathrm{O}_{2}\right)$ for $15 \mathrm{~min}$ at approximately $80{ }^{\circ} \mathrm{C}$ and dried with $\mathrm{N}_{2}$, before a UV/ozone treatment, as previously used. ${ }^{32}$

Neutron Reflectometry (NR). NR is a powerful tool for measuring the composition of material at buried interfaces, particularly in layers perpendicular to a surface or interface (e.g., solid-liquid interface). The neutron beam can penetrate through some solid materials $\left(\mathrm{Al}_{2} \mathrm{O}_{3}\right.$ in this case $)$ and is either reflected or refracted at each interface, depending on the wavelength, incident angle, and difference in refractive indices of the materials either side of the interface. This leads to constructive and destructive interference in the reflected signal (Kiessig fringes). The theoretical details can be found elsewhere. ${ }^{33-35}$ In this study, NR is used to measure the interference between bulk $\mathrm{Al}_{2} \mathrm{O}_{3}$ and water as well as the adsorbed surfactant AOT at the interface. $\mathrm{D}_{2} \mathrm{O}$ is used as the bulk water phase as it has a similar scattering length density (related to refractive index) to $\mathrm{Al}_{2} \mathrm{O}_{3}$, but very different than AOT. Therefore, this maximizes the contrast and hence sensitivity to the AOT in the system. When there is a structure of repeating layers, Bragg peaks will also appear in the reflected signal. In this case, these are observed from the repeating bilayers of $\mathrm{AOT}^{23}$

In this paper, the NR measurements were carried out at the ILL neutron source on the FIGARO beamline. ${ }^{36}$ A rheometer was placed on the sample stage, and the neutron beam reflected up through the solid substrate to the solid-liquid interface (see schematic in Figure 1). Each incident angle provides a range of neutron wavelengths and therefore a range of momentum transfer, $Q_{z}(=4 \pi / \lambda \sin \theta)$. Two incident angles of $0.375^{\circ}$ and $1.8^{\circ}$ were used to generate the full reflectivity profile. To observe the changes in scattering with time under shear, only the higher angle was used due to the location of the Bragg peaks in this $Q$ range. A resolution of $7 \%$ was used for the lower concentration measurements and improved to $5 \%$ for the Bragg peak measurements. The sample stage was kept close to horizontal during the measurements by using a supermirror to alter the incoming beam angle to $0.617^{\circ}$ and $1.4^{\circ}$ for the two incident angles. The footprint was kept at $35 \times 35 \mathrm{~mm}$ and centered beneath the cone: smaller than the $50 \mathrm{~mm}$ diameter circle under the rheometer cone. GISANS measurements were also taken on the same instrument by altering the beam collimation, with a footprint of $7 \times 45 \mathrm{~mm}$.

The data were reduced in the usual way using the COSMOS program within LAMP. ${ }^{37}$ The background was subtracted using adjacent pixels on the 2D detector away from any off-specular scattering. The data were fitted using LAMP and Rascal (A. Hughes, ISIS). The GISANS images were reduced using an IDL procedure provided by Erik Watkins (Los Alamos National Laboratory, USA), which was also used to convert the detector images to $Q$-space when considering changes in off-specular scattering. 
Rheology. An Anton Paar MCR501 Physica rheometer was used with a Ti cone attachment, of angle $1.0^{\circ}$ and central gap size 0.097 $\mathrm{mm}$, and a diameter of $50 \mathrm{~mm}$. The alumina crystal was clamped in place on the bottom plate of the rheometer. A solvent trap was used to try and minimize evaporation/H-D exchange during the measurements. The sample stage was set to a constant temperature of $12{ }^{\circ} \mathrm{C}$, which was monitored with a thermocouple between the crystal and aluminum mount. The measured sample temperature averaged $18{ }^{\circ} \mathrm{C}$ throughout the experiment.

Initial measurements of the high concentration AOT showed a very slight shear alignment at $0.01 \mathrm{~s}^{-1}$. Therefore, this preshear was applied to all high concentration measurements outlined here to provide a constant sample history. Shear rates up to $5 \mathrm{~s}^{-1}$ and $1000 \%$ at $10 \mathrm{~Hz}$ were applied until no change was observed in the reflectivity (approximately $1 \mathrm{~h}$ ).

\section{RESULTS AND DISCUSSION}

Neutron Data (Low Concentration Surfactant). NR measurements were taken for $2.5 \mathrm{mM}$ AOT solution on alumina from $\mathrm{D}_{2} \mathrm{O}$ under static conditions. A large fringe was observed in the data compared to the bare $\mathrm{Al}_{2} \mathrm{O}_{3}-\mathrm{D}_{2} \mathrm{O}$ interface (Figure 2), corresponding to an adsorbed layer of

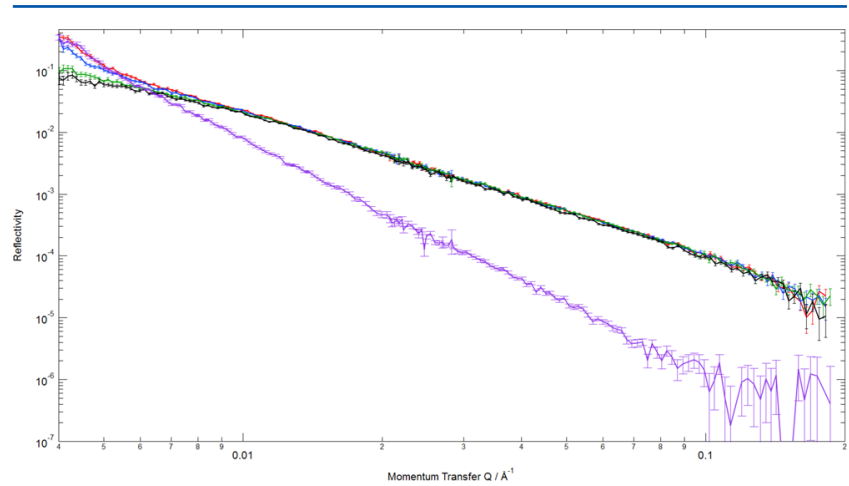

Figure 2. Reflectivity curves of $2.5 \mathrm{mM}$ AOT in $\mathrm{D}_{2} \mathrm{O}$ on alumina under increasing steady shear rates from 0.5 to $500 \mathrm{~s}^{-1}$. The bare $\mathrm{D}_{2} \mathrm{O}-\mathrm{Al}_{2} \mathrm{O}_{3}$ curve is shown for comparison (purple). The drop in critical edge is due to $\mathrm{H}-\mathrm{D}$ exchange.

AOT at the interface. The data are fitted to a model consisting of a symmetric head-tail-tail-head bilayer, with fittable parameters for the projected area per molecule (APM) and water molecules per head. These parameters then provide the scattering length density (SLD) profile, using known volumes for the head and tail portions of the molecule and conserving the head:tail ratio. The variation in SLD essentially provides the changes in composition away from the interface and is given by the sum of the neutron scattering lengths from all atoms within a unit cell divided by the volume of that unit cell. The structural parameters of Hellsing et al. $^{22}$ are used and show a good agreement, indicating that the previously reported bilayer has been recreated in this work. This corresponds to an APM of 57 $\AA^{2}$ and 12 water molecules per headgroup, yielding a total thickness of $37 \AA$.

A slightly low SLD for $\mathrm{D}_{2} \mathrm{O}$ of $6.2 \times 10^{-6} \AA^{-2}$ was required to fit the critical edge, corresponding to a small amount (approximately 2 vol \%) of $\mathrm{H} / \mathrm{D}$ exchange of the $\mathrm{D}_{2} \mathrm{O}$ in this relatively open sample setup. The same sample was measured repeatedly with time, and the low $Q$ region showed a small shift in the critical edge $\left(Q_{z}\right.$ below which complete external reflection is observed) consistent with this exchange. This is just observed at the low $Q$ limit in Figure 2. Because of the relative SLDs within this system, the critical edge is particularly sensitive to these small changes. It was not possible to stop this small level of exchange within this setup, despite significant efforts to prevent this. The rest of the reflectivity curve is not significantly affected over the experimental time scales.

Having established the static structure, the behavior of the adsorbed bilayer under steady and dynamic shear was determined. Figure 2 shows the reflectivity curves for the surfactant in $\mathrm{D}_{2} \mathrm{O}$ increasing steady shear rates from 0.5 to 500 $\mathrm{s}^{-1}$. The only significant change occurs at low $Q$ near the critical edge, consistent with the H/D exchange discussed above. Therefore, we conclude that shear rates up to $500 \mathrm{~s}^{-1}$ have no effect on the adsorbed AOT bilayer at the aluminawater interface at $2.5 \mathrm{mM}$.

The same result was found for applied oscillatory shear up to $200 \%$ at $100 \mathrm{~Hz}$. We therefore conclude that oscillatory shear does not affect the surfactant bilayer structure under these shear conditions.

These experimental results are significant as they contradict some "folklore" that flow in pipes can disrupt protective additive layers. These results show that either the layer is completely unaffected or any removed surfactant is replenished on the experimental time scale. This may be due to a stationary layer of fluid near the surface under this shear (boundary layer) or because the attractive adsorption forces are stronger than the shear forces. NR is particularly sensitive to changes in the layer thickness, which would identify changes in the tilt of molecules within the bilayer. Given that no change was observed, this suggests that the surface layer does not move at all (i.e., there is laminar flow, with a stationary boundary layer at least $37 \AA$ thick, bilayer thickness).

We now describe similar experiments at a higher concentration of AOT, which shows longer range ordering from the surface and may be more susceptible to imposed shear.

Neutron Data (High Concentration Data). Interpreting the Changes under Shear. Figure 3 shows an example set of reflectivity curves for $50 \mathrm{mM}$ AOT adsorbed on $\mathrm{Al}_{2} \mathrm{O}_{3}$ under a constant shear rate of $1 \mathrm{~s}^{-1}$ over time. Two Bragg peaks are present within the measured $Q$-range at approximately 0.03 and $0.06 \AA^{-1}$. This is attributed to the formation of a number of surfactant bilayers aligned parallel to the sapphire surface. The surfactant layer spacing can be calculated from the $d$-spacing of

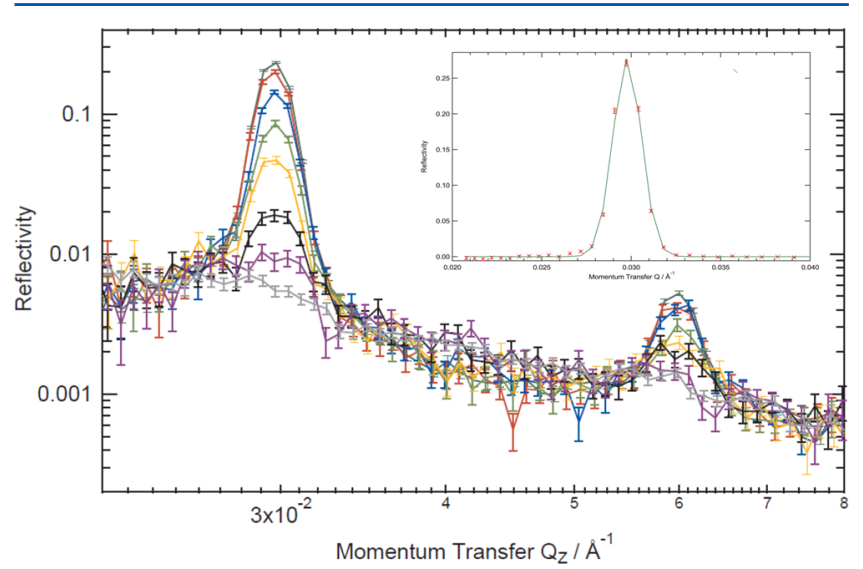

Figure 3. A series of reflectivity profiles measured at the higher angle of $1.8^{\circ}$ under and applied steady shear rate of $1 \mathrm{~s}^{-1}$, with $50 \mathrm{mM}$ AOT at the alumina $-\mathrm{D}_{2} \mathrm{O}$ interface. The data sets were measured in $5 \mathrm{~min}$ intervals. Inset shows a typical Gaussian fit (line) to the experimental data (crosses). 

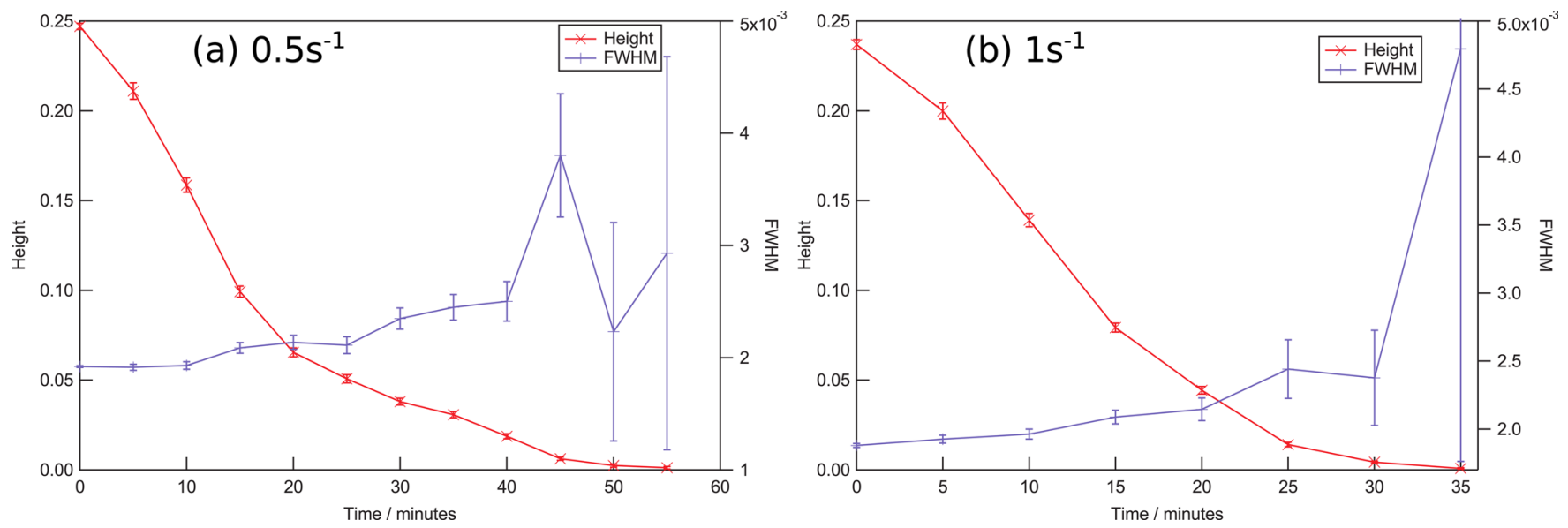

Figure 4. Example variations in Bragg peak height (red cross) and fwhm (blue plus) for samples under steady shear of (a) $0.5 \mathrm{~s}^{-1}$ and (b) $1 \mathrm{~s}^{-1}$. Each data point is determined from a single run and plotted at the time corresponding to its end.

the Bragg peaks giving a spacing of $209 \AA$, in good agreement with previous literature. ${ }^{22,23,25,26,38}$ This corresponds to the total thickness of the surfactant bilayer $(\sim 37 \AA)$ and a water spacer layer $(\sim 172 \AA)$ between.

Under static conditions these features remain indefinitely, but the two Bragg peaks show a reduction in intensity under shear. Each data set was recorded for 5 min to allow sufficient quality data to resolve the peaks, but the data between the peaks remains noisy. As the system is constantly changing, each run shows the average reflectivity from the structure over the 5 min period. As both of these peaks occur in data collected at a single reflection angle, measurements at this single reflection angle alone have been used to probe the structural variations over time.

The changes with time at fixed shear rate can be characterized as follows: there is a reduction in the peak intensity and possibly an increase in the peak width. After approximately $40 \mathrm{~min}$ the peaks were lost completely. A longer neutron measurement (with applied shear) for improved statistics showed no change to the large Kiessig fringe from the first AOT bilayer adsorbed to the alumina surface when compared to the static measurement. Hence, the applied shear has removed the ordered multilayers at the surface leaving the single adsorbed bilayer found at the CMC. This loss of multilayer adsorption is consistent with the suggestion of Thomas and Penfold that the AOT multilayers formed at the air-water interface adsorb relatively weakly. ${ }^{24}$ At this concentration the bulk phase contains lamellar crystallites, which become ordered in the vicinity of the surface. This is partially due to the "templating" of the crystallites by the initial surface bilayer adsorbed onto the alumina surface. However, this concentration is relatively close to the boundary with the micellar phase in the bulk phase diagram, which facilitates the breakdown of the multilayer ordering near the surface, as per the discussions of Thomas and Penfold. ${ }^{24}$

Interpretation of the Bragg Scattering. It is found that Bragg peaks within the reflectivity data change under applied shear. The Bragg peaks fall within the Kiessig fringe from the AOT bilayer at the surface, which creates a nonlinear baseline to the peak. To simplify the fitting process and minimize the number of model parameters, the scattering is considered as the sum of the surface scattering (solid-liquid interface and adsorbed AOT bilayer) and scattering from the multilayers (Bragg peaks). Therefore, the reflectivity of the "final" state, where only the scattering from the surface bilayer remains, can be used as a background and subtracted. The resultant curve consists of only the Bragg peaks with a simple linear background. This follows a similar argument to that used by Hellsing et al. ${ }^{23}$ and Penfold et al. ${ }^{39}$ In this work we adopt an analysis based on the peak heights and widths of each peak separately as the patterns change under shear. These parameters are not expected to be greatly dependent on the approaches used to remove a background contribution.

The "final" state of the $1 \mathrm{~s}^{-1}$ experiment was used as background. This was particularly important for the oscillatory shear samples for which the peaks were not fully lost. The use of other "final state" steady shear runs produced essentially the same result.

The first Bragg peak has a much greater intensity and occurs further away from the background scattering level than the Bragg peak at higher $Q$ making it the most suitable peak for quantitative analysis. The resultant peaks were then fitted to a standard Gaussian distribution, with a typical fit shown in Figure 3. In each case this peak was fitted over a $Q$-range of $0.02-0.04 \AA^{-1}$. Analysis with a pseudo-Voigt distribution also provided a good fit to the peaks, with the same overall trends observed.

The widths of the diffraction peaks can have a number of origins which mainly include the instrumental resolution, finite size effects (from a limited number of bilayers in the stack), and paracrystalline/strain structural distortions in the interbilayer distances. If we assume the broadening is from a purely finite size origin, we can use the Scherrer equation, to estimate the number of ordered layers at the interface from the peak width (after an allowance for the experimental resolution). Using this model, the presheared sample has approximately 20 ordered bilayers at the interface, which compares well to that of Hellsing et al. 23

We have also considered a "Williamson-Hull" plot where the broadening of peaks (after subtraction of the experimental resolution) of different order (001), (002), (003), etc., can be used to deduce the relative contributions of finite size and positional disorder between the layers. This analysis might suggest that there is a significant component of paracrystalline distortion in the bilayer stacks. However, in this work we cannot confidently confirm the origin of the peak broadening in this way. There are only two peaks, one of which is relatively low in intensity and the extent of broadening above the 

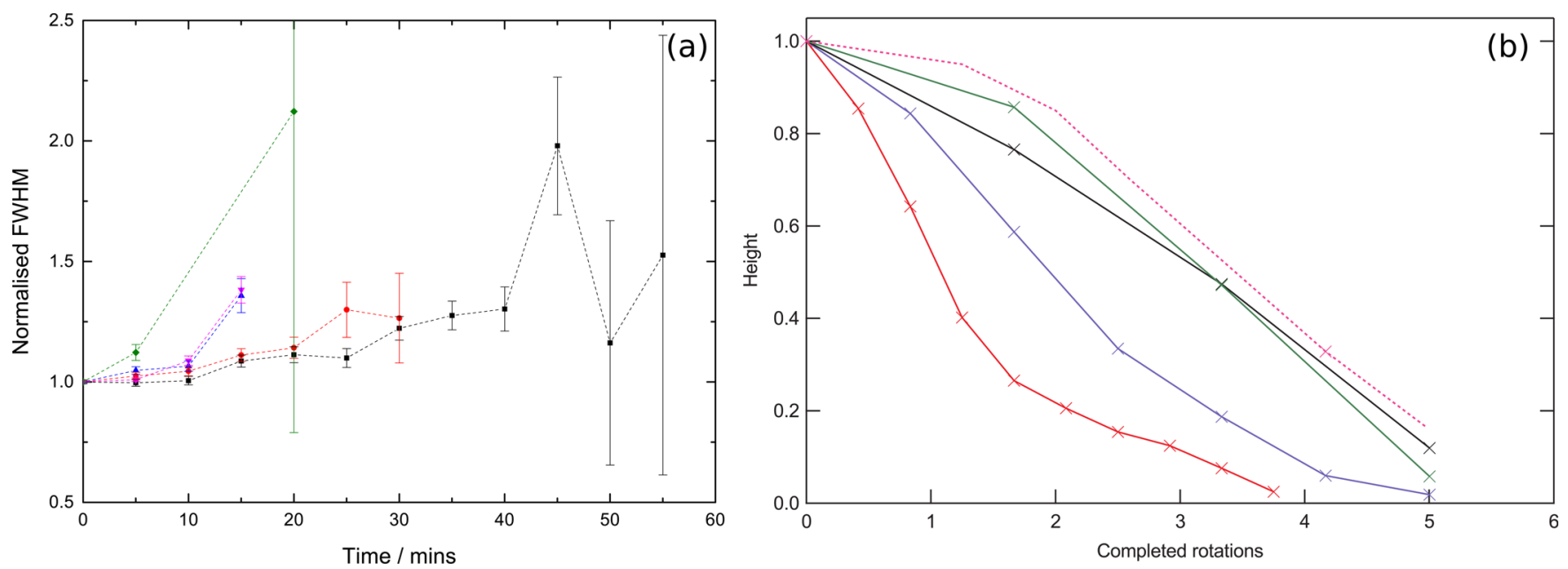

Figure 5. (a) Summary of the normalized Bragg peak fwhm with time for the different applied steady shear rates. Black $=0.5 \mathrm{~s}^{-1}$, red $=1 \mathrm{~s}^{-1}$, blue $=$ $2 \mathrm{~s}^{-1}$, pink $=2 \mathrm{~s}^{-1}$ (repeat), green $=5 \mathrm{~s}^{-1}$. Lines are a guide to the eye. (b) Summary of the Bragg peak height with completed rotations at each steady shear rate. The heights have been normalized to the preshear value. Red $=0.5 \mathrm{~s}^{-1}$, blue $=1 \mathrm{~s}^{-1}$, black $=2 \mathrm{~s}^{-1}$, green $=2 \mathrm{~s}^{-1}$ (repeat), pink $=5$ $\mathrm{s}^{-1}$. The lines are a guide to the eye, and the $5 \mathrm{~s}^{-1}$ shows a dashed expected trend given the lack of measured points.
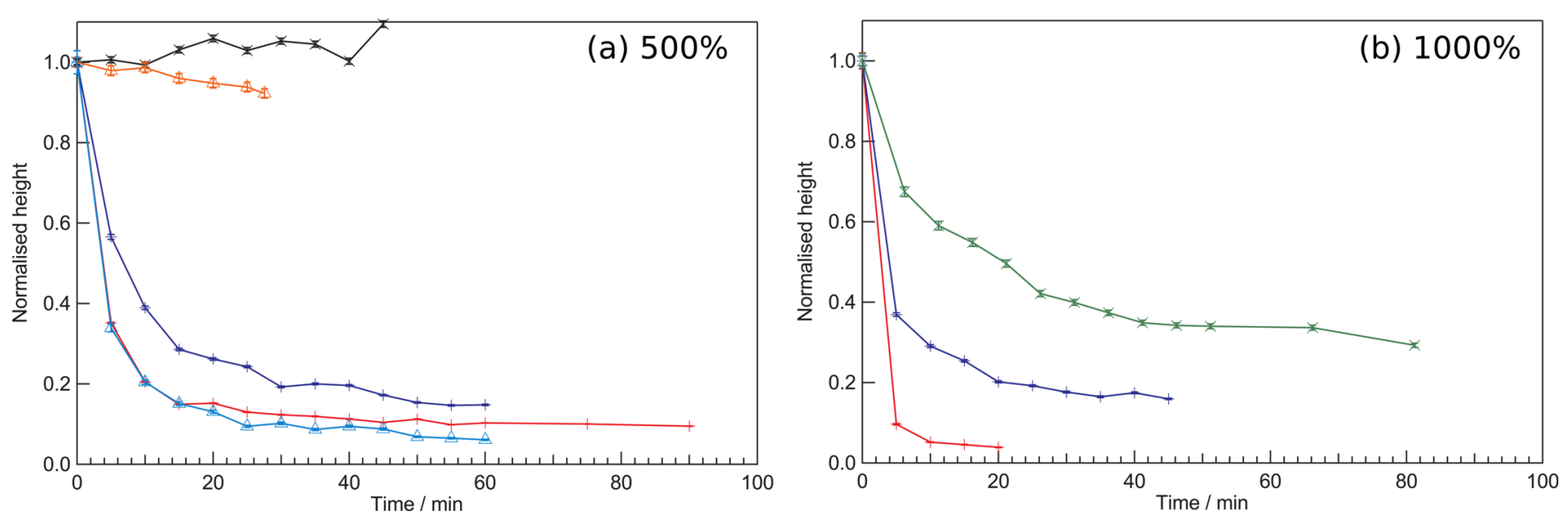

Figure 6. Summary of the height variation within the Bragg peak under oscillatory shear amplitudes of $500 \%$ and $1000 \%$, with varying frequency. (a) Black cross $=1 \mathrm{~Hz}$; orange triangle $=2 \mathrm{~Hz}$; blue plus $=5 \mathrm{~Hz}$; red plus $=10 \mathrm{~Hz}$; light blue triangle $=20 \mathrm{~Hz}$. (b) Green cross $=1 \mathrm{~Hz}$; blue plus $=5$ $\mathrm{Hz}$; red cross $=10 \mathrm{~Hz}$. Each data set is normalized to the preshear peak height.

experimental resolution is modest. Hence, our analysis focuses on the changes of the peak width of the most intense peak only and, although attributed to finite size effects, may well have a significant or dominant contribution from paracrystalline distortion. It is the change in peak intensity that is most dramatic.

In each case, the height of the Bragg peak decreased with time accompanied by a slight increase in width, while the peak position remained constant. The peak height/intensity is a complex function of the contrasts, hydration, and number of layers. The peak center defines the repeat distance. Therefore, these changes indicate that the number of layers in the multilayer decreases slightly under applied shear but the structure/repeat distance of the remaining layers stays the same. These variations will now be considered in more detail over a range of shear conditions.

Steady Shear. Steady shear measurements were made at $0.01,0.02,0.05,0.5,1,2$, and $5 \mathrm{~s}^{-1}$ as a series of $5 \mathrm{~min}$ neutron runs. For the lowest three shear rates, no change in structure was observed after $40 \mathrm{~min}$ and within the experimental error. However, for shear rates above $0.05 \mathrm{~s}^{-1}$, a steady drop in the Bragg peak intensity was observed, with complete loss of intensity after a characteristic time. Figure 3 shows an example of the changes in the reflectivity, and Figures $4 \mathrm{a}$ and $4 \mathrm{~b}$ show an example of the variation in peak heights and widths with time for shear rates of 0.5 and $1 \mathrm{~s}^{-1}$, respectively. A steady decay in the peak height can be seen in the figure until the peak can no longer be discerned. This suggests that the amount of this phase/structure is decreasing and essentially disappears: either as a lower fraction across the surface or as a reduction in the number of multilayers. The decrease in height is accompanied by a small increase in the peak width with time. As the peak disappears, the error in the fitted width becomes very large but clearly represents a small broadening. This suggests the loss in peak intensity may be coupled to a reduction in the number of layers or a loss of structural correlations between the layers. There is no significant change in the peak position as the intensity drops, suggesting that the spacing between layers does not change and that the layers are removed steadily possibly with some component of a delamination type process, but more significantly a reduction in the total amount of lamellar phase present at the surface. Similar trends in the scattering were found at 2 and $5 \mathrm{~s}^{-1}$. 
Figure 5a shows the variation in the fwhm for each shear rate normalized to the preshear value. While shearing, the initial loss of structure appears to follow a similar trend for all steady shear rates. This loss appears to be initially quite slow, followed by rapid loss of the remaining order in each case at a "characteristic" time. At this point the peak widths can no longer be accurately fitted as they have become so broad and shallow. This common initial loss suggests that the long-range order is removed by a common mechanism, with the "characteristic" time decreasing as the shear rate increases.

To further compare the different shear rates, the variation in peak height was considered as a function of the effective distance traveled by the disc (the applied strain). The data are shown in Figure $5 \mathrm{~b}$ and show a dramatic drop in peak height, with the Bragg peak indistinguishable from the bilayer scattering at a similar applied strain amplitude. This strain is approximately five rotations or a strain amplitude (shear rate $X$ time) of approximately 1800 . This is a relatively high strain amplitude but is comparable to the values observed for MLV formation at higher surfactant and/or salt concentrations for $\mathrm{AOT}^{3}$ and other surfactant mixtures. ${ }^{40}$

Oscillatory Shear. For the dynamic shear experiments two strain amplitudes were used (500\% and $1000 \%)$ over a range of frequencies from 1 to $20 \mathrm{~Hz}$. These measurements follow the same procedure as for the steady shear experiments.

The lowest shear rates of 1 and $2 \mathrm{~Hz}$ at 500\% showed no loss in the peak intensity, with possibly a small increase in intensity (shear ordering). However, significant changes in the scattering were observed at frequencies above $5 \mathrm{~Hz}$, suggesting there is a critical value for structural change between 2 and $5 \mathrm{~Hz}$ at 500\%. Figure 6 shows the variation in normalized peak height with time for each of the applied shear conditions. It can be seen that applying an oscillatory shear produced a drop in the Bragg peak intensity to a new equilibrium structure, represented by a plateau in the peak intensity, rather than a complete loss of the feature. This contrasts with the steady shear results and suggests that a critical strain is needed for complete removal. The figure also shows that the peak height falls more quickly and to a lower plateau value as the frequency of oscillation or the amplitude is increased. The Bragg peak was not completely removed under any of the applied oscillatory conditions, which suggests differences in how the system is affected by steady and oscillatory shear.

A couple of shear ramps were also completed. Once the $500 \%, 5 \mathrm{~Hz}$ data had reached a plateau, 10 and $20 \mathrm{~Hz}$ were then applied in turn; and when the $500 \%, 10 \mathrm{~Hz}$ measurement had equilibrated, 50 and $100 \mathrm{~Hz}$ were applied. An additional loss of intensity was observed between the 5 and $10 \mathrm{~Hz}$ measurements as well as a small loss between the 10 and $50 \mathrm{~Hz}$; however, these plateaued to a slightly lower peak height than for the individual measurement. The higher frequencies did not appear to have a significant effect. This is in reasonable agreement with the individual changes shown in Figure $6 \mathrm{a}$ and suggests that the trends in loss are not strongly dependent on shear history.

Figure 7 shows the variation in the peak fwhm normalized to the preshear value for each oscillatory shear condition. In each case there appears to be a rise in this width followed by a plateau value, as for the peak height. In each case, the center position of the Bragg peak is constant.

Comparison of Steady vs Dynamic Shear. In summary, it was found that an applied steady shear of $0.5 \mathrm{~s}^{-1}$ or higher completely removed the long-range order in an AOT multilayer after a characteristic period of time (i.e., complete loss of the

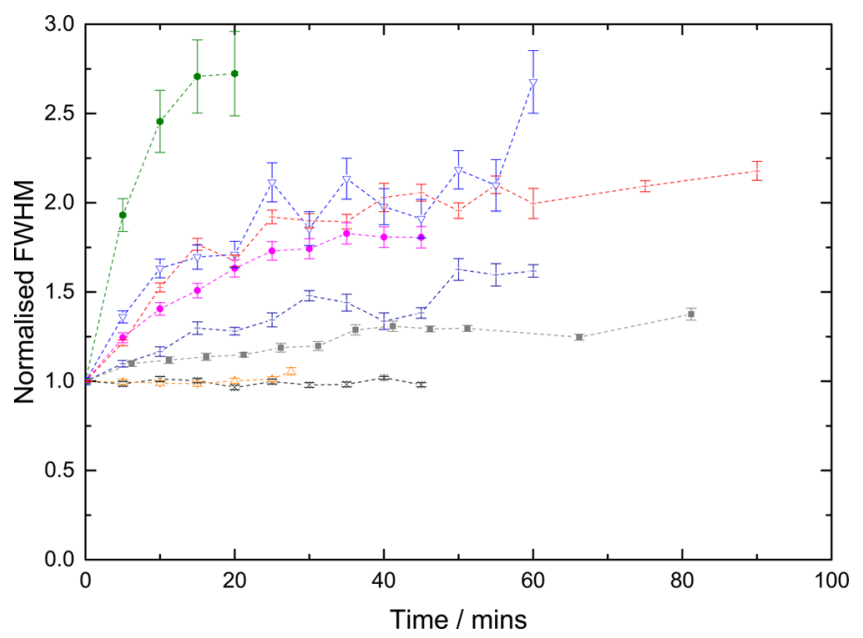

Figure 7. Variation in the normalized peak width under oscillatory shear. For the $500 \%$ amplitude: black cross $=1 \mathrm{~Hz}$; orange triangle $=2$ $\mathrm{Hz}$; blue plus $=5 \mathrm{~Hz}$; red plus $=10 \mathrm{~Hz}$; light blue triangle $=20 \mathrm{~Hz}$. For the $1000 \%$ amplitude: gray filled triangle $=1 \mathrm{~Hz}$; pink filled square $=5 \mathrm{~Hz}$; green filled circle $=10 \mathrm{~Hz}$.

multilayer structure to leave only the surface bilayer). Below $0.05 \mathrm{~s}^{-1}$ no change of structure was observed on the experimental time scale. The characteristic time was dependent on the shear rate applied-a reflection of the particular strain amplitude.

The oscillatory shear also showed a loss in long-range order, but interestingly only to another equilibrium structure. The final value of the intensity was dependent on the shear rate applied, in contrast to steady shear for which the initial loss appeared to be independent of the applied shear rate. Again there is some evidence of line broadening which may suggest a delamination mechanism or increased paracrystalline distortion in both cases. Within these studies, amplitudes of 500 and $1000 \%$ were used for the dynamic measurements. These both fall below the critical strain amplitude of 1800, which was estimated from the steady shear data and may help explain the observed differences. If a higher amplitude was applied during the dynamic measurements, the Bragg peaks may have also been completely lost.

These differences can also be considered in regards to the motion of the fluid. The transition between laminar and turbulent flow, as well as the thickness of any stationary boundary layer, is dependent on the ratio of the inertial to viscous forces and so varies with fluid velocity (Reynold's number). During a steady shear experiment a constant velocity is applied throughout, whereas the velocity will vary sinusoidally during a dynamic shear experiment. This means that, on average, during a dynamic experiment the Reynold's number will be smaller. This also may help explain the observed increased stability of the multilayer under dynamic shear.

Loading the Sample. During the experiment, it was observed that the method of loading the sample was important in producing repeatable multilayers. Therefore, the method was kept consistent throughout all the experiments presented within this paper, including the volume of liquid used, height the cone was lowered from, and the speed of the cone when lowered into place. As an extreme comparison, the sample well was overfilled and the cone lowered into place through this liquid (compared to only adding a small volume under the cone). In this case, only small Bragg peaks were observed 

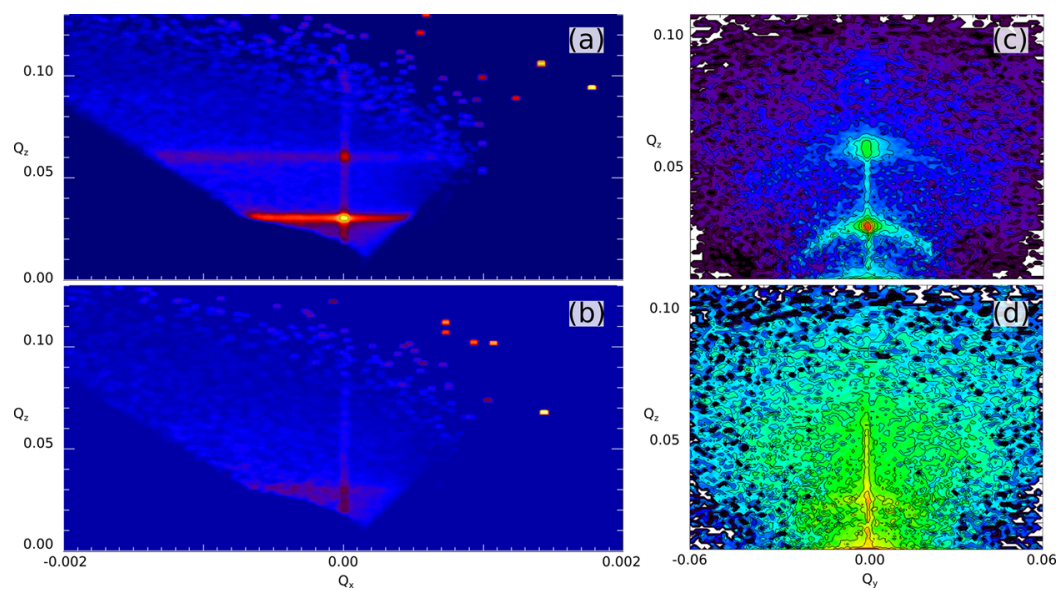

Figure 8. Off-specular scattering data: (a) $Q_{x} Q_{z}$ map of NR data before shear; (b) $Q_{x} Q_{z}$ map of NR data after shear; (c) $Q_{y} Q_{z}$ map of GISANS data before shear; (d) $Q_{y} Q_{z}$ map of GISANS data after shear.

within the reflectivity, which occurred at a slightly higher $Q$. This indicates a decrease in the spacing between layers. It is hypothesized that the "squeezing" as the cone is lowered induces a stress within the liquid parallel to the surface, as it wets the alumina substrate, which enhances the lamellar ordering at the surface. If the cell is already full then, the surfactant solution already covers the alumina surface and is bound by the solid walls of the O-ring. In this case the stress parallel to the surface is not large enough to induce the same ordering as the surface is already in contact with the lamellar phase.

In-Plane Behavior. In addition to the specular reflectivity observations, some further information about the multilayers can be obtained by considering the off-specular scattering. Offspecular scatter indicates that there is correlation of scattering events within the sample plane from structural order in these directions $\left(Q_{x}\right.$ and $\left.Q_{y}\right)$. In the data here, significant off-specular scattering was found around each Bragg peak, as shown in Figures $8 \mathrm{a}, \mathrm{b}$, which is consistent with previous observations for lamellar surfactant systems and is generally assigned to correlated thermal undulations of the multilayers. ${ }^{23-26,38}$

In this present work, when shear was applied, the intensity of this off-specular scatter was found to fall. The off-specular intensity appears to be lost at a slightly quicker rate than the specular Bragg peak, which may suggest that the lamellar sheets are broken up or "flattened" during the shearing process, i.e., causing more rapid loss in the correlated undulations than the peak intensity.

Two GISANS measurements were taken before and after applying a steady shear of $2 \mathrm{~s}^{-1}$, at a glancing angle of $1.4^{\circ}$ and wavelength band of $2-20 \AA$, with the data shown in Figures $8 \mathrm{c}$,d. GISANS probes structure within the first $10 \mu \mathrm{m}$ of the solution from the surface and provides additional information about in-plane ordering. Figure $8 \mathrm{c}$ shows a weak powder pattern about each intense Bragg peak, which indicates an isotropic arrangement of domains within the plane with some preferential orientation parallel to the interface. This is consistent with thermal undulations which would be expected to occur in all directions within the plane. After applying a shear rate of $2 \mathrm{~s}^{-1}$ the order within the GISANS measurement was lost (Figure $8 \mathrm{~d}$ ), with a broadening of the peaks and some possible damping of the bilayer oscillations, in agreement with the specular and off-specular Bragg peak observations.
The combination of Bragg peaks and powder pattern suggests a preferential ordering of lamellar layers very close to the surface, which become randomly orientated a couple of micrometers into the bulk. (Both surface and near-surface contributions are included in these measurements.) This is consistent with both the previously reported lamellae at the surface and the crystallites of lamellar phase formed in bulk solution.

\section{CONCLUSION}

The behavior of an anionic surfactant under applied shear at the solid-liquid interface has been measured at two concentrations: around the $\mathrm{cmc}(2.5 \mathrm{mM})$ and just into the lamellar phase $(50 \mathrm{mM})$. The low concentration forms a bilayer at the interface, which is not altered by the applied steady or dynamic shear. This indicates that adsorbed layers of around $37 \AA$ are not altered by typical pipe-flow regimes. These shear rates fall within the hydrodynamic region of the Stribeck curve, where loads are relatively low and adsorbed layers can form on the surfaces. These layers are suggested to effectively move the shear plane away from the solid surface, providing some lubrication. For a bilayer this may be to the outer face or to the middle of the tail region. At much higher loads (boundary regime) the solid surfaces start to come into contact (tribology and contact asperities) and lubrication breaks down, which is beyond the scope of this current work.

The higher concentration surfactant solution forms multilayers which align on the surface and propagate out into the solution and which are lost under applied shear (above a critical rate). Under steady shear the multilayers are lost completely, whereas under dynamic shear a new equilibrium structure with either fewer repeating layers or more paracrystalline structural disorder is observed. This new structure is dependent on both the frequency and amplitude of the applied oscillation. In both cases the surface bilayer is not lost. By considering off-specular scattering, the in-plane correlations are also lost, with no MLVs forming close to the interface. When combined with the rheology and visible observations, this suggests that the bulk lamellar phase is no longer stable at these shear rates (above $0.05 \mathrm{~s}^{-1}$ or $500 \%, 2 \mathrm{~Hz}$ ) at $50 \mathrm{mM} \mathrm{AOT}$, which is relatively close to the micellar-phase boundary.

The point of loss is found to be dependent on the strain amplitude under steady shear. This is similar to the critical strain previously observed for MLV formation (at higher AOT/ 
$\mathrm{NaCl}$ concentrations). This could suggest there is a similar effect on the lamellar stability here, but there is insufficient surfactant (or charge screening) to form MLVs at this lower concentration, water-continuous part of the lamellar region of the phase diagram.

\section{ASSOCIATED CONTENT}

\section{S Supporting Information}

The Supporting Information is available free of charge on the ACS Publications website at DOI: 10.1021/acs.langmuir.7b00530.

Discussion of the rheology measurements from the neutron reflectivity experiment and additional offline rheology measurements (PDF)

\section{AUTHOR INFORMATION}

\section{Corresponding Author}

*E-mail: becky.welbourn@stfc.ac.uk (R.J.L.W.).

\section{ORCID $\odot$}

Rebecca J. L. Welbourn: 0000-0002-4254-5354

Stuart M. Clarke: 0000-0001-5224-2368

\section{Present Address}

R.J.L.W.: ISIS, Rutherford Appleton Laboratory, Harwell Science and Innovation Campus, Didcot, UK.

\section{Notes}

The authors declare no competing financial interest.

\section{ACKNOWLEDGMENTS}

We thank BP plc [grant number EP/J501876/1] and the Engineering and Physical Sciences Research Council, UK (EPSRC) for financial support for this work as well as the ILL staff and scientists for the allocation of beam time and technical assistance with NR measurements.

\section{REFERENCES}

(1) Diat, O.; Roux, D.; Nallet, F. Effect of Shear on a Lyotropic Lamellar Phase. J. Phys. II 1993, 3 (9), 1427-1452.

(2) Diat, O.; Roux, D.; Nallet, F. Layering" effect in a Sheared Lyotropic Lamellar Phase. Phys. Rev. E: Stat. Phys., Plasmas, Fluids, Relat. Interdiscip. Top. 1995, 51 (4), 3296-3300.

(3) Bergenholtz, J.; Wagner, N. J. Formation of AOT/Brine Multilamellar Vesicles. Langmuir 1996, 12, 3122-3126.

(4) Leon, A.; Bonn, D.; Meunier, J.; Al-Kahwaji, A.; Greffier, O.; Kellay, H. Coupling between Flow and Structure for a Lamellar Surfactant Phase. Phys. Rev. Lett. 2000, 84 (6), 1335-1338.

(5) Leon, A.; Bonn, D.; Meunier, J. Transitions Induced by Shear in Surfactant Phases and Relaxation of the Shear-Induced Phases. J. Phys.: Condens. Matter 2002, 14, 4785-4794.

(6) Panizza, P.; Roux, D.; Vuillaume, V.; Lu, C.; Cates, M. Viscoelasticity of the Onion Phase. Langmuir 1996, 12 (7), 248-252. (7) Adlmann, F. A.; Gutfreund, P.; Ankner, J. F.; Browning, J. F.; Parizzi, A.; Vacaliuc, B.; Halbert, C. E.; Rich, J. P.; Dennison, A. J. C.; Wolff, M. Towards Neutron Scattering Experiments with SubMillisecond Time Resolution. J. Appl. Crystallogr. 2015, 48 (1), $220-226$.

(8) Wolff, M.; Steitz, R.; Gutfreund, P.; Voss, N.; Gerth, S.; Walz, M.; Magerl, A.; Zabel, H. Shear Induced Relaxation of Polymer Micelles at the Solid-Liquid Interface. Langmuir 2008, 24 (20), 11331-11333.

(9) Wolff, M.; Akgun, B.; Walz, M.; Magerl, A.; Zabel, H. Slip and Depletion in a Newtonian Liquid. EPL 2008, 82 (3), 36001.

(10) Gutfreund, P.; Wolff, M.; Maccarini, M.; Gerth, S.; Ankner, J. F.; Browning, J.; Halbert, C. E.; Wacklin, H.; Zabel, H. Depletion at Solid/liquid Interfaces: Flowing Hexadecane on Functionalized Surfaces. J. Chem. Phys. 2011, 134 (6), 064711.
(11) Corvis, Y.; Barré, L.; Jestin, J.; Gummel, J.; Cousin, F. Asphaltene Adsorption Mechanism under Shear Flow Probed by in Situ Neutron Reflectivity Measurements. Eur. Phys. J.: Spec. Top. 2012, 213 (1), 295-302.

(12) Penfold, J.; Staples, E.; Tucker, I.; Fragnetto, G. The Effect of Shear on the Adsorption of Non-Ionic Surfactants at the Liquid-Solid Interface. Phys. B 1996, 221, 325-330.

(13) van der Grinten, D.; Wolff, M.; Zabel, H.; Magerl, A. Flow Cell for Grazing Incidence Neutron Scattering. Meas. Sci. Technol. 2008, 19 (3), 034016.

(14) Morgan, C. E.; Breward, C. J. W.; Griffiths, I. M.; Howell, P. D.; Penfold, J.; Thomas, R. K.; Tucker, I.; Petkov, J. T.; Webster, J. R. P. Kinetics of Surfactant Desorption at an Air-Solution Interface. Langmuir 2012, 28 (50), 17339-17348.

(15) Hamilton, W. A.; Butler, P. D.; Magid, L. J.; Han, Z.; Slawecki, T. M. Fast Relaxation of a Hexagonal Poiseuille Shear-Induced nearSurface Phase in a Threadlike Micellar Solution. Phys. Rev. E: Stat. Phys., Plasmas, Fluids, Relat. Interdiscip. Top. 1999, 60, R1146-R1149.

(16) Hamilton, W. A.; Porcar, L.; Magid, L. J. Using Neutron Reflectometry and Reflection Geometry "near-Surface" SANS to Investigate Surfactant Micelle Organization at a Solid-solution Interface. Phys. B 2005, 357 (1-2), 88-93.

(17) Newby, G. E.; Watkins, E. B.; Merino, D. H.; Staniec, P. A.; Bikondoa, O. In Situ Rheo-GISANS of Triblock Copolymers: Gelation and Shear Effects on Quasi-Crystalline Structures at Interfaces. RSC Adv. 2015, 5 (126), 104164-104171.

(18) Graca, M.; Bongaerts, J. H. H.; Stokes, J. R.; Granick, S. Friction and Adsorption of Aqueous Polyoxyethylene (Tween) Surfactants at Hydrophobic Surfaces. J. Colloid Interface Sci. 2007, 315 (2), 662-670.

(19) Kuhl, T. L.; Smith, G. S.; Israelachvili, J. N.; Majewski, J.; Hamilton, W. Neutron Confinement Cell for Investigating Complex Fluids. Rev. Sci. Instrum. 2001, 72 (3), 1715.

(20) de Vos, W. M.; Mears, L. L. E.; Richardson, R. M.; Cosgrove, T.; Dalgliesh, R. M.; Prescott, S. W. Measuring the Structure of Thin Soft Matter Films under Confinement: A Surface-Force Type Apparatus for Neutron Reflection, Based on a Flexible Membrane Approach. Rev. Sci. Instrum. 2012, 83 (11), 113903.

(21) de Vos, W. M.; Mears, L. L. E.; Richardson, R. M.; Cosgrove, T.; Barker, R.; Prescott, S. W. Nonuniform Hydration and Odd-Even Effects in Polyelectrolyte Multilayers under a Confining Pressure. Macromolecules 2013, 46 (3), 1027-1034.

(22) Hellsing, M. S.; Rennie, A. R.; Hughes, A. V. Effect of Concentration and Addition of Ions on the Adsorption of Aerosol-OT to Sapphire. Langmuir 2010, 26 (18), 14567-14573.

(23) Hellsing, M. S.; Rennie, A. R.; Hughes, A. V. Adsorption of Aerosol-OT to Sapphire: Lamellar Structures Studied with Neutrons. Langmuir 2011, 27 (8), 4669-4678.

(24) Thomas, R. K.; Penfold, J. Multilayering of Surfactant Systems at the Air-Dilute Aqueous Solution Interface. Langmuir 2015, 31 (27), $7440-7456$

(25) Li, Z. X.; Weller, A.; Thomas, R. K.; Rennie, A. R; Webster, J. R. P.; Penfold, J.; Heenan, R. K.; Cubitt, R. Adsorption of the Lamellar Phase of Aerosol-OT at the Solid/Liquid and Air/Liquid Interfaces. J. Phys. Chem. B 1999, 103, 10800-10806.

(26) Li, Z. X.; Lu, J. R.; Thomas, R. K.; Penfold, J. Neutron Specular and off-Specular Reflection from the Surface of Aerosol-OT Solutions above the Critical Micelle Concentration. Faraday Discuss. 1996, 104, 127-138.

(27) Rogers, J.; Winsor, P. A. Change in the Optic Sign of the Lamellar Phase (G) in the Aerosol OT/water System with Composition or Temperature. J. Colloid Interface Sci. 1969, 30 (2), 247-257.

(28) Kumar, S. K. K.; Tamimi, A.; Fayer, M. D. Dynamics in the Interior of AOT Lamellae Investigated with Two-Dimensional Infrared Spectroscopy. J. Am. Chem. Soc. 2013, 135 (13), 5118-5126.

(29) Valdes, M.; Manero, O.; Soltero, J. F. A.; Puig, J. E. Rheology of Lyotropic Liquid Crystals of Aerosol OT 1. Low Concentration Regime. J. Colloid Interface Sci. 1993, 160, 59-64. 
(30) Robles-Vásquez, O.; Corona-Galvan, S.; Soltero, J. F. A.; Puig, J. E.; Tripodi, S. B.; Valles, E.; Manero, O. Rheology of Lyotropic Liquid Crystals of Aerosol OT 2. High Concentration Regime. J. Colloid Interface Sci. 1993, 160, 65-71.

(31) Robles-Vásquez, O.; Soltero, J. F. A.; Puig, J. E.; Manero, O. Rheology of Lyotropic Liquid Crystals of Aerosol OT 3. Effect of Salt and Hydrocarbons. J. Colloid Interface Sci. 1994, 163, 432-436.

(32) Welbourn, R. J. L.; Lee, S. Y.; Gutfreund, P.; Hughes, A.; Zarbakhsh, A.; Clarke, S. M. Neutron Reflection Study of the Adsorption of the Phosphate Surfactant NaDEHP onto Alumina from Water. Langmuir 2015, 31 (11), 3377-3384.

(33) Penfold, J.; Thomas, R. K. The Application of the Specular Reflection of Neutrons to the Study of Surfaces and Interfaces. J. Phys.: Condens. Matter 1990, 2, 1369-1412.

(34) Penfold, J.; Richardson, R. M.; Zarbakhsh, A.; Webster, J. R. P.; Bucknall, D. G.; Rennie, A. R.; Jones, R. A. L.; Cosgrove, T.; Thomas, R. K.; Higgins, J. S.; Fletcher, P. D. I.; Dickinson, E.; Roser, S. J.; Mclure, I. A.; Hillman, A. R.; Richards, R. W.; Staples, E. J.; Burgess, A. N.; Simister, E. A.; White, J. W. Recent Advances in the Study of Chemical Surfaces and Interfaces by Specular Neutron Reflection. J. Chem. Soc., Faraday Trans. 1997, 93 (22), 3899-3917.

(35) Pynn, R. In Neutron Applications in Earth, Energy and Environmental Sciences; Liang, L., Rinaldi, R., Schober, H., Eds.; Neutron Scattering Applications and Techniques; Springer: Boston, MA, 2009.

(36) Campbell, R. A.; Wacklin, H. P.; Sutton, I.; Cubitt, R.; Fragneto, G. FIGARO: The New Horizontal Neutron Reflectometer at the ILL. Eur. Phys. J. Plus 2011, 126 (11), 107.

(37) Large Array Manipulation Program; www.ill.eu/data_treat/ lamp/the-Lamp-Book.

(38) Li, Z. X.; Lu, J. R.; Thomas, R. K.; Weller, A.; Penfold, J.; Webster, J. R. P.; Sivia, D. S.; Rennie, A. R. Conformal Roughness in the Adsorbed Lamellar Phase of Aerosol-OT at the Air - Water and Liquid - Solid Interfaces. Langmuir 2001, 17, 5858-5864.

(39) Penfold, J.; Tucker, I.; Thomas, R. K.; Taylor, D. J. F.; Zhang, J.; Zhang, X. L. The Impact of Electrolyte on the Adsorption of Sodium Dodecyl Sulfate/Polyethyleneimine Complexes at the Air-Solution Interface. Langmuir 2007, 23, 3690-3698.

(40) Escalante, J. I.; Gradzielski, M.; Hoffmann, H.; Mortensen, K. Shear-Induced Transition of Originally Undisturbed Lamellar Phase to Vesicle Phase. Langmuir 2000, 16 (23), 8653-8663. 